\title{
Tailoring the chirality of light emission with spherical Si-based antennas
}

\author{
Xavier Zambrana-Puyalto* and Nicolas Bonod ${ }^{\dagger}$ \\ Aix-Marseille Université, CNRS, Centrale Marseille, \\ Institut Fresnel UMR 7249, 13013 Marseille, France
}

Chirality of light is of fundamental importance in several enabling technologies with growing applications in life science, chemistry and photodetection. Recently, some attention is being focused on chiral quantum emitters. Consequently, optical antennas which are able to tailor the chirality of light emission are needed. Spherical nanoresonators such as colloids are of particular interest to design optical antennas since they can be synthesized at large scales and they exhibit good optical properties. Here, we show that these colloids can be used to tailor the chirality of a chiral emitter. To this purpose, we derive an analytic formalism to model the interaction between a chiral emitter and a spherical resonator. We then compare the performances of metallic and dielectric spherical antennas to tailor the chirality of light emission. It is seen that, due to their strong electric dipolar response, metallic spherical nanoparticles spoil the chirality of light emission by yielding achiral fields. In contrast, thanks to the combined excitation of electric and magnetic modes, dielectric Si-based particles feature the ability to inhibit or to boost the chirality of light emission. Finally, it is shown that dual modes in dielectric antennas preserve the chirality of light emission. 


\section{INTRODUCTION}

Optical antennas can resonantly interact with light to enhance the decay rates and to control the directivity of emission of quantum emitters, or reciprocally to enhance the near fields. They have been successfully

used for example in molecular spectroscopy [1, 2], single photon sources [3-5] and photo-detectors [6]. A lot of efforts have also been devoted to design optical antennas which are able to enhance chiral lightmatter interactions. Among recent achievements, we find enhancing the resolution of the circular dichroism technique [7]; controlling the optical rotation of light [8]; creating chiral optical fields from linearly polarized plane waves $[9,10]$; creating complex chiral plasmonic structures [11-15]; or even digging in the interplay between the angular momentum (AM) of light and chirality [16-22].

Here we investigate the interaction between a chiral dipolar emitter $[23,24]$ and a spherical optical antenna. Emphasis is placed on spherical nanoparticles since they can be fabricated at large scales via colloidal synthesis or laser ablation with excellent surface and optical properties [25-29]. The question that we address is whether individual spherical nanoparticles can be used to produce strong chiral effects when interacting with chiral emitters. More precisely, we aim at studying how the antenna modifies the chirality of the emitted field in the far-field region. For that purpose, we present a simple formalism to analyze the chirality of the scattered light. This formalism, which is analytic and valid for antennas of any size, is developed using the Mie theory in the helicity basis [30-32]. The formalism is first applied to plasmonic antennas composed by gold spherical nanoparticles [33-35], and secondly to dielectric spherical resonators made of silicon $[28,29,36-38]$. It will be demonstrated that the interplay between electric and magnetic modes of the optical antennas is required to tailor the chirality of light in the far field. In addition, it will be seen that the dual behavior of the particle [30, 31, 39] plays a very important role.

\section{CHIRAL DIPOLAR EMISSION}

At the level of macroscopic Maxwell equations, we consider that a dipolar emitter $\mathcal{C}$ is chiral if and only if its emission is described as a superposition of both electric and magnetic dipolar fields. In contrast, we 
consider that the emitter is non-chiral if its emission pattern is symmetric under at least one mirror symmetry plane. In particular, the field described by either either an electric or a magnetic dipolar field alone are nonchiral. It is also assumed that an emitter whose emitted electromagnetic (EM) field is a combination of electric and magnetic dipolar fields has both electric and magnetic dipole moments, $\mathbf{p}$ and $\mathbf{m}$. Note that for chiral emitters, $\mathbf{p}$ must be a real magnitude, and $\mathbf{m}$ must be imaginary [40][41, p187].

The description of the EM fields in terms of electric and magnetic multipoles has been proven to be very useful for a huge variety of phenomena. However, for chirality and optical activity studies [42-44], a change from the electric/magnetic (or parity) basis to the helicity $+1 /-1$ basis $[32,45]$ allows for an easier description of the problem. The change from the parity to the helicity basis is made in the following way. Let $\left\{\mathbf{A}_{j m_{z}}^{(e)}, \mathbf{A}_{j m_{z}}^{(m)}\right\}$ be electric and magnetic multipoles. Then, their linear combinations yield multipolar fields of well-defined helicity [30, 32, 46]:

$$
\mathbf{A}_{j m_{z}}^{+}=\frac{\mathbf{A}_{j m_{z}}^{(m)}+i \mathbf{A}_{j m_{z}}^{(e)}}{\sqrt{2}} \quad \mathbf{A}_{j m_{z}}^{-}=\frac{\mathbf{A}_{j m_{z}}^{(m)}-i \mathbf{A}_{j m_{z}}^{(e)}}{\sqrt{2}}
$$

As a result, $\mathbf{A}_{j m_{z}}^{+}$and $\mathbf{A}_{j m_{z}}^{-}$are eigenstates of the helicity operator $\Lambda[39,46]$ :

$$
\Lambda \mathbf{A}_{j m_{z}}^{+}=+\mathbf{A}_{j m_{z}}^{+} \quad \Lambda \mathbf{A}_{j m_{z}}^{-}=-\mathbf{A}_{j m_{z}}^{-}
$$

Notice that for both families of multipoles, the sub-indexes $j, m_{z}$ stand for the eigenvalues of the operators $\left(J^{2}\right)$ and $\left(J_{z}\right)[32]$. Now, in the same way that a current density $\mathbf{j}$ can be given by an electric or magnetic dipolar moment ( $\mathbf{p}$ or $\mathbf{m})$, it can also be given by a helicity \pm 1 dipolar moment $\left(\mathbf{p}_{+}\right.$or $\left.\mathbf{p}_{-}\right)$[47].

In Figure 1, we show the emitted fields of the emitter $\left|\mathbf{E}^{\mathcal{C}}\right|^{2}$ for two different orientations of $\mathbf{p}_{+}$and $\mathbf{p}_{-}$ corresponding to $m=0,+1$ (or alternatively $\hat{\mathbf{z}}$ and $(\hat{\mathbf{x}}+i \hat{\mathbf{y}}) / \sqrt{2}$ ). As it can be observed, the radiation pattern of $\mathbf{p}_{+}$and $\mathbf{p}_{-}$for $m=1$ are counter-intuitive, as they are highly directional. This contrasts with the highly non-directional radiation patterns of electric and magnetic dipoles. Nonetheless, the pattern can be inferred from the definition of helicity and its relation to the AM of light [30, 39].

Then, we will define the chirality $(C)$ of the emitter as the comparative strength of $\mathbf{p}_{+}$and $\mathbf{p}_{-}$. That is, if the emitted field by the emitter is given by $\mathbf{E}^{\mathcal{C}}=\alpha \mathbf{A}_{1, m_{z}}^{+}+\sqrt{1-|\alpha|^{2}} \mathbf{A}_{1, m_{z}}^{-}$, then we will define $C$ as:

$$
C(\alpha)=\frac{|\alpha|^{2}-\left(1-|\alpha|^{2}\right)}{|\alpha|^{2}+\left(1-|\alpha|^{2}\right)}=2|\alpha|^{2}-1
$$



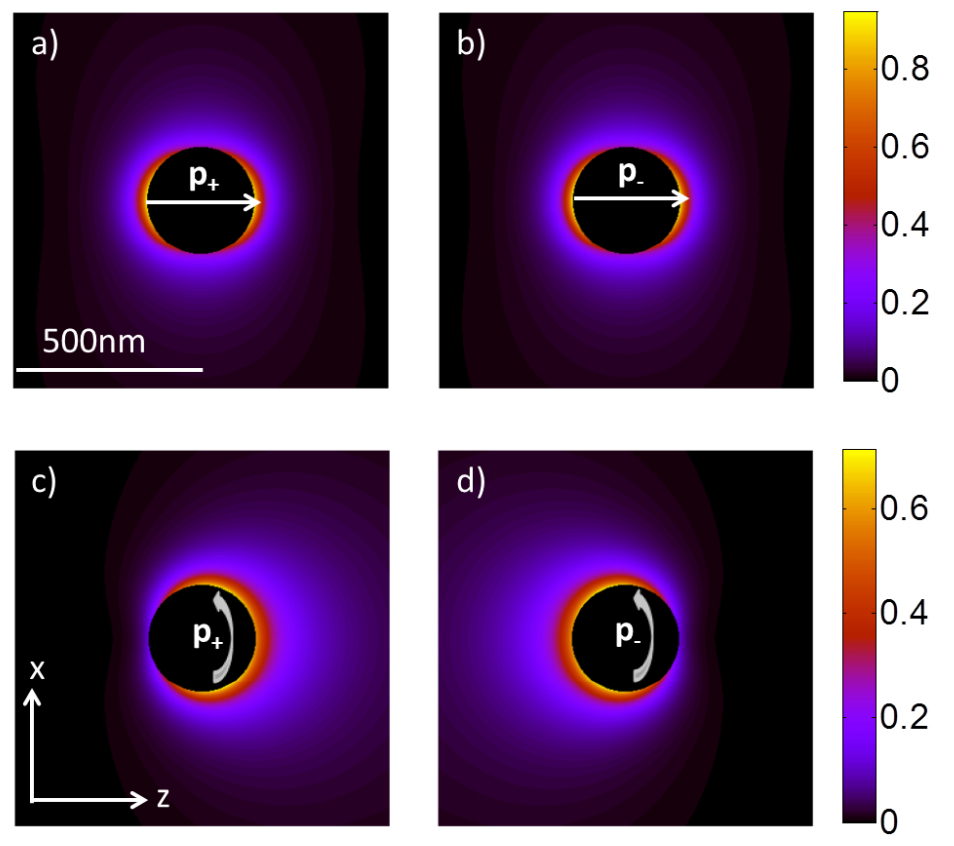

FIG. 1. $\left|\mathbf{E}^{\mathcal{C}}\right|^{2}$ of an emitting dipolar emitter. For a) and b) the dipole is oriented along the $z$ axis, whereas for c) and $\mathrm{d})$ it is left circularly oriented, i.e. $\mathbf{p}_{ \pm} \propto(\hat{\mathbf{x}}+i \hat{\mathbf{y}})$. The dipole emits light with helicity +1 in a) and $\left.\mathrm{c}\right)$, and with helicity -1 in b) and d). The emission is at $\lambda=632 \mathrm{~nm}$. A null field has been assumed in a spherical volume of radius $100 \mathrm{~nm}$ to remove the divergence of dipolar fields at the origin. Each image is $1 \mu \mathrm{m} \times 1 \mu \mathrm{m}$ in size.

where $|\alpha|^{2}$ is the probability that the emitter shines light with helicity +1 . As a result, the probability that the emitter emits light with helicity -1 is $\left(1-|\alpha|^{2}\right)$. Using the definition given by eq.(3), the chirality of the emitter $C(\alpha)$ will be a value going from $[-1,+1]$. It can be seen that $C(\alpha)=+1$ indicates that the emitter emission is only given by $\mathbf{p}_{+} ; C(\alpha)=0$ means that the probability of inducing $\mathbf{p}_{+}$and $\mathbf{p}_{-}$is the same - this is the case of an emitter which is purely given by $\mathbf{p}$ or $\mathbf{m}$; finally, $C(\alpha)=-1$ indicates that the emission is only given by $\mathbf{p}_{-}$. The same definition of chirality will be used to quantify the chirality of the scattered light in the far field. 


\section{MULTIPOLAR FORMALISM TO ANALYZE THE CHIRALITY OF THE SCATTERED FIELDS}

In this section, we provide analytic tools to easily describe the chirality of the field scattered off a spherical antenna when the latter is coupled to a chiral emitter. Without any loss of generality, it is assumed that the chiral emitter is located in the $z$ axis and emits light at a unitary rate. We suppose that the chirality of the emitter, given by $C(\alpha)$, is an inherent property. Then, we analytically compute the chirality of the scattered field $\left(C^{s}(\alpha)\right)$ as a function of $\alpha$, the distance between the emitter and the antenna, and the size of the antenna. The theoretical details to compute $C^{s}(\alpha)$ are given in Appendix A, but the general idea can be summarized as follows. The chiral source emits a dipolar field which is a superposition of two dipolar fields with opposite well-defined helicities: $\mathbf{E}^{\mathcal{C}}=\alpha \mathbf{A}_{1, m_{z}}^{+}+\sqrt{1-|\alpha|^{2}} \mathbf{A}_{1, m_{z}}^{-}$, where $m_{z}$ has to do with the orientation of the emitter. The reference frame of the problem is not located at the center of the emitter, but rather at the center of the antenna. Thus, the translation-addition formulas need to be used to find an analytic expression of the emitted chiral field. Then, the Mie theory in the helicity basis [30-32] is used to find the scattered field $\mathbf{E}^{\mathrm{s}}$. This scattered field has, in general, two helicity components which contribute separately to the total scattered power. That is, the total scattered power $W^{\mathrm{s}}$ is computed as $W^{\mathrm{s}}=W_{+}^{\mathrm{s}}+W_{-}^{\mathrm{s}}$, with $\mathrm{W}_{+}^{\mathrm{s}}$, and $\mathrm{W}_{-}^{\mathrm{s}}$ being the scattered power into modes with helicity +1 and -1 , respectively. Then, following the definition of $C(\alpha)$ given by eq.(3), we compute $C^{s}(\alpha)$ as:

$$
C^{s}(\alpha)=\frac{\mathrm{W}_{+}^{\mathrm{s}}-\mathrm{W}_{-}^{\mathrm{s}}}{\mathrm{W}_{+}^{\mathrm{s}}+\mathrm{W}_{-}^{\mathrm{s}}}
$$

As a result, the chirality of the scattered field by the antenna can be analytically expressed as (see Appendix A):

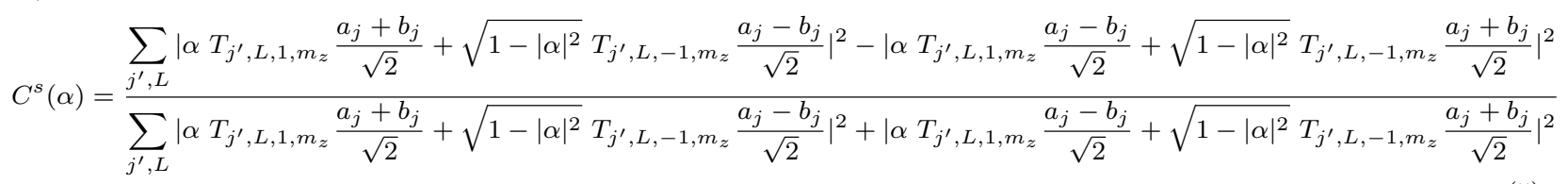

Note that $C^{s}(\alpha)$ is a multi-variable function and depends upon different parameters apart from $\alpha$. Naturally,

it depends on the features of the emitter such as its emitting wavelength $\lambda_{0}$, its orientation $m_{z}$, and its distance to the particle $r_{0}$. In addition, $C^{s}(\alpha)$ depends on some parameters of the antenna such as its radius $R$, its permittivity $\epsilon$ and permeability $\mu$. 

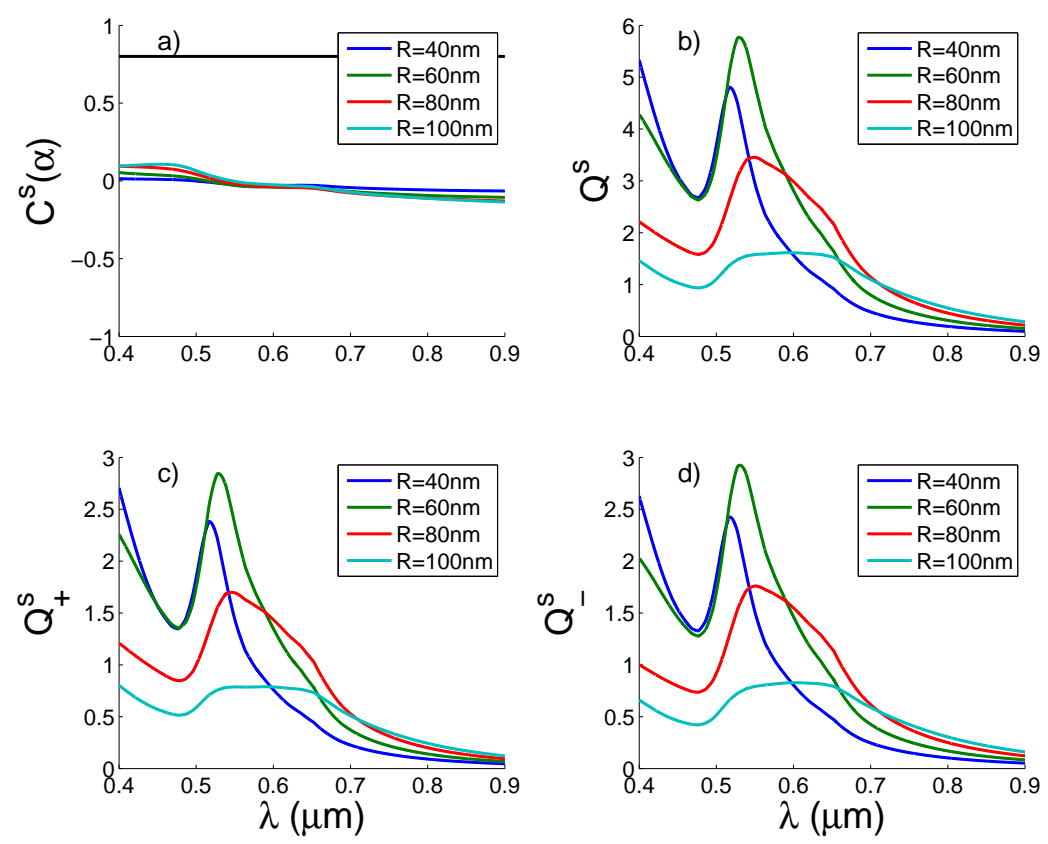

FIG. 2. a) Scattered far-field chirality signal. The black straight line represents $C(\alpha=\sqrt{0.9})$, whereas the colored lines display $C^{s}(\alpha=\sqrt{0.9}, R)$ for $R=40,60,80,100 \mathrm{~nm}$. b) Scattering efficiencies $\left(Q^{s}\right)$ for the same 4 antennas. c) Scattering efficiency factor $\left(Q_{+}^{s}\right)$ corresponding to a helicity +1 state. d) Scattering efficiency factor $\left(Q_{-}^{s}\right)$ corresponding to a helicity -1 state. The same color code is used for all plots. All the multipolar contributions of the antenna are taken into account for all calculations. The antenna is made of $\mathrm{Au}$.

\section{GOLD PLASMONIC ANTENNA}

Let us first study the chirality properties of the field scattered by a plasmonic antenna made of gold, with $\mu=1$ and $\epsilon$ given by a linear interpolation of the data points in [48]. Four different radius of spherical antennas will be considered, $R=40,60,80,100 \mathrm{~nm}$. We consider a chiral dipolar emitter with $|\alpha|^{2}=0.9$, emitting from the visible to the near-infrared $\lambda \in[400,900] \mathrm{nm}$, and located at a constant distance of $10 \mathrm{~nm}$ along the $z$ axis from the surface of the gold particle. The orientation of the emitter for both helicity components is assumed to be along the $z$ axis, i.e. $\mathbf{p}_{+}=p_{+} \hat{\mathbf{z}}$, and $\mathbf{p}_{-}=p_{-} \hat{\mathbf{z}}$. This orientation passes on to eq.(5) as $m_{z}=0$. It has been numerically verified that this choice of orientation does not affect the results in a qualitative manner. 
The spectra of $C^{s}(\alpha, R)$ are displayed in Fig.2a) for the four different particle diameters. Each of the four subplots of Fig.2a) displays $C^{s}(\sqrt{0.9}, R)$ as well as the initial chirality of the emitter, $C(\sqrt{0.9})=0.8$. It is observed that the chiral behavior of the antenna is almost flat in the whole spectrum and that $C^{s}(\sqrt{0.9}, R) \sim$ 0 for the four sizes and for the whole spectral range. Moreover, this behavior is completely independent of the resonant behavior of the particle. In Fig.2b), we plot the efficiency factors $Q^{s}$ [49] and observe that despite the different spectral resonances in $Q^{s}$ depicted in Fig.2b), their chirality behavior is almost identical and constant to $C^{s}(\sqrt{0.9}, R) \sim 0$. Now, due to the definition of $C^{s}(\alpha)$ (see eq.(4)), this implies that both helicity components of the scattered field are almost equal. Actually, this feature could have been predicted beforehand, as it is well known that a gold nanoparticle approximately behaves as an electric dipole. Now, an electric dipole has a 50\% - 50\% decomposition into dipolar fields of two different helicities [50, 51]. This fact can be directly deduced from eq.(1). Hence, it is clear that an electric dipolar particle such as a gold nanoparticle is not a good candidate to enhance the far-field chirality signal of a chiral light emitter.

Figures 2c) and d) show the scattering efficiency factor $Q^{s}$ split into its two helicity components, $Q^{s}=$

$Q_{+}^{s}+Q_{-}^{s}$ (see Appendix A). That is, if we add the values from the plots in Figs.2c) and d), we obtain the plots of Figure 2b). Because the nanoantenna almost behaves as an electric dipole, it can be observed that $Q_{+}^{s}$ and $Q_{-}^{s}$ almost yield the same result. Nonetheless, the unitary emission of the chiral emitter, which is modeled by a black line both in Figure 2c) and d), is mostly done (with a probability of $|\alpha|^{2}=0.9$ ) in the +1 helicity state; the emission in the -1 helicity state happens with a probability of $\left(1-|\alpha|^{2}=0.1\right)$. Hence, the nanoantenna clearly increases the relative strength of the helicity -1 component, whereas it decreases the strength of the other component. In other words, what a gold nanoantenna does is transforming a big amount of the main helicity state $(+1$ for this case) of the incident light into the opposite helicity state $(-1$ in this example).

\section{SI-BASED MIE ANTENNA}

We now aim at studying whether Si-based spherical particles hosting electric and magnetic modes are a valuable alternative to plasmonic antennas for tailoring chiral light emission. In Fig.3 (in blue, left y-axis), we 

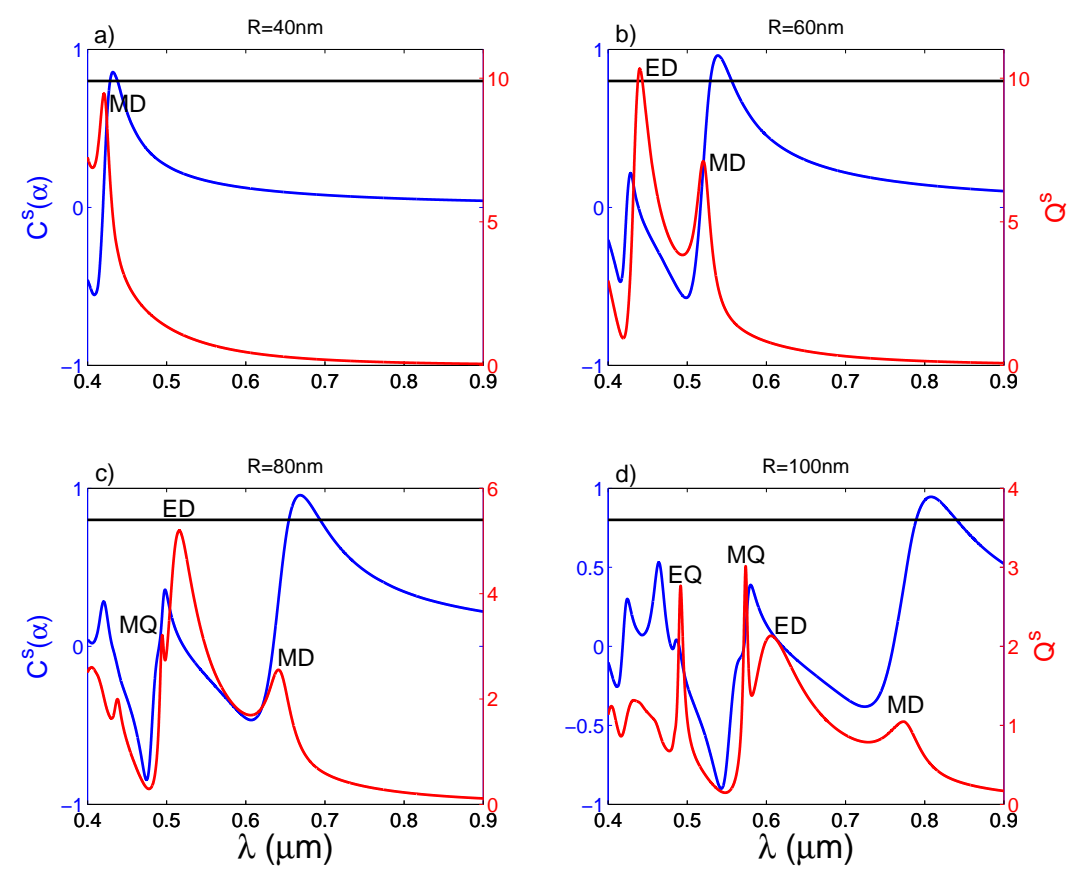

FIG. 3. In blue, plots of the scattered far-field chirality $C^{s}(\alpha=\sqrt{0.9}, R)$. In black, the initial chirality of the emitter $C(\alpha=\sqrt{0.9})$. In red, the scattering efficiency factor $Q^{s}$. Each subplot a)b)c)d) corresponds to a different size of the resonator given by $R=40,60,80,100 \mathrm{~nm}$. The resonator is made of Si. All the multipolar contributions have been taken into account for all the plots. The electric/magnetic dipolar and quadrupolar contributions have been explicitly marked. The chiral emitter has $|\alpha|^{2}=0.9$, it is at a $10 \mathrm{~nm}$ distance from the surface of the antenna along the $z$ axis, oriented along the $z$ axis, i.e. $m_{z}=0$. The permittivity of Si has been obtained from [52].

plot $C^{s}(\alpha)$ for a Si nanoantenna of radius $R=40,60,80,100 \mathrm{~nm}$ using Eq.(5). The properties of the emitter are the same as those used in the previous section. Note that even though this choice of sizes might seem restrictive, it is demonstrated in Appendix B that Si-based antennas achieve their full potential chirality within this regime. The chirality response of the Si nanoantenna displayed in Fig.3 contrasts with the rather constant behavior observed with gold particles. It can be observed that coupling a chiral emitter with a spherical silicon particle almost covers the whole range of chirality values for the EM field: the antenna can boost, preserve or even invert the chirality of the emitted fields. However, even if the antenna offers all these possibilities, the scattered power is not constant throughout the spectral range. For this reason, we have also 

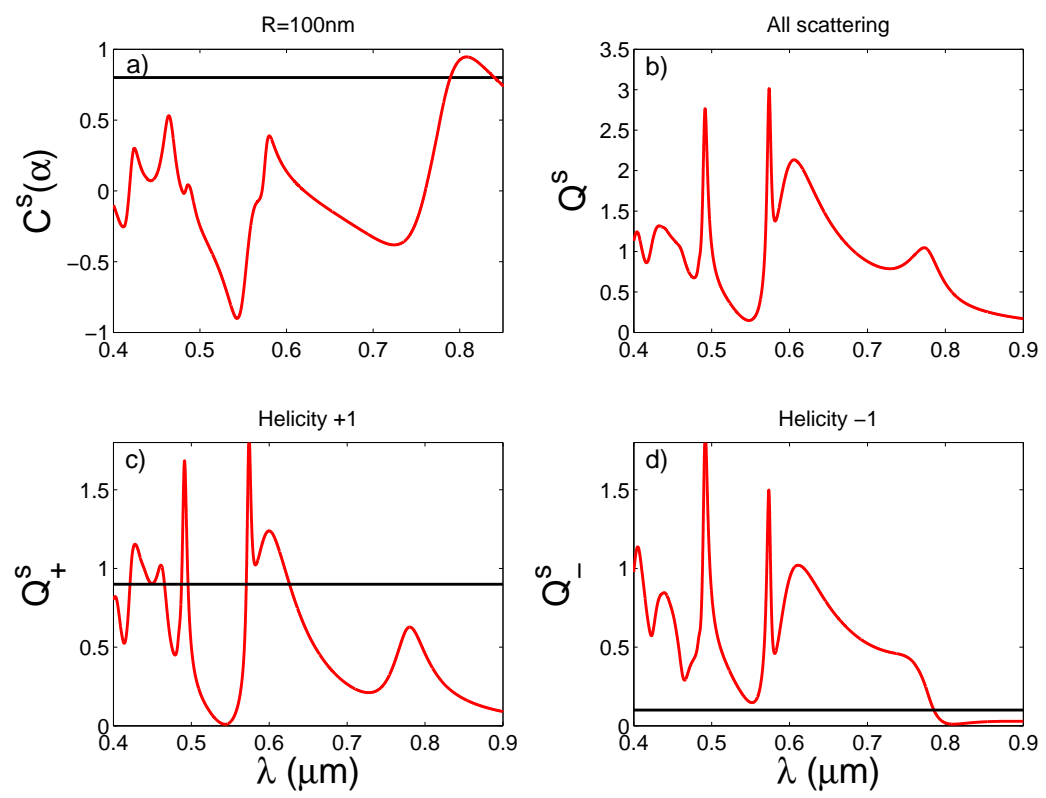

FIG. 4. A chiral dipolar emitter with $|\alpha|^{2}=0.9$ in the $\lambda=400-900 \mathrm{~nm}$ range interacts with an antenna of radius $R=100 \mathrm{~nm}$ made of Si. a) $C^{s}(\alpha=\sqrt{0.9})$ in red and $C(\alpha=\sqrt{0.9})$ in black. b) Scattering efficiency factor $Q^{s}$ for the antenna. c) Helicity +1 component of $Q^{s}\left(Q_{+}^{s}\right)$ in red, and the probability of the emitter to emit in a helicity +1 state $\left(|\alpha|^{2}=0.9\right)$ in black. d) Helicity -1 component of $Q^{s}\left(Q_{-}^{s}\right)$ in red, and the probability of the emitter to emit in a helicity -1 state $\left(1-|\alpha|^{2}=0.1\right)$ in black.

depicted $Q^{s}$ in Fig.3 (in red, right y-axis). In particular, it is seen that the spectral position of the absolute minimum of $C^{s}(\alpha)$ always coincides with a local minimum of $Q^{s}$. This effect is specially prominent for the particles with $R=80,100 \mathrm{~nm}$. The underlying reason is an almost null scattered field $\mathbf{E}^{\mathrm{s}}$ in these two cases. Hence, due to the definition of $C^{s}(\alpha)$ given by Eq.(4), small variations of the helicity components $\mathrm{W}_{+}^{\mathrm{s}}$ and $\mathrm{W}_{-}^{\mathrm{s}}$ around a zero value can certainly account for great variations. Notice that when $C^{s}(\alpha)<0$ and specially when $C^{s}(\alpha) \sim-C(\alpha)$, the antenna mostly scatters light with the opposite chirality. This was defined in [30-32] as an anti-dual behavior. However, we observe that this non-trivial behavior comes with a price: the scattered power is highly reduced.

Another interesting feature of these Si-based antennas is that they can reach $C^{s}(\alpha) \simeq 1$. That is, they can almost scatter all the light in only one helicity component. To unveil the physical process, we plot in 
Fig.4 $C^{s}(\alpha), Q^{s}, Q_{+}^{s}$ and $Q_{-}^{s}$ for a Si-based antenna of $R=100 \mathrm{~nm}$. The other parameters are the same as they were in Fig.3. For Fig.4c)d), a black straight line is plotted to represent the probability to observe light emitted in a \pm 1 helicity state. Now, Fig.4d) unveils that the absolute maximum of $C^{s}(\alpha)$ occurs for a non-trivial minima of the initial small helicity component (the - component for this example). That is, $C^{s}(\alpha)$ yields the purest chiral state when the energy scattered in the - helicity component is minimum.

It is also worth commenting that, even though the emitter emits with a probability of $\left(|\alpha|^{2}=0.9\right)$ in the +1 helicity component, and with a probability $\left(1-|\alpha|^{2}=0.1\right)$ in the -1 component, $Q_{+}^{s}$ and $Q_{-}^{s}$ have a similar spectral behavior (see Fig.4c) and d)). The underlying reason is that the resonances of a dielectric Mie antenna are of either electric or magnetic nature, and electric/magnetic modes split $50 \%-50 \%$ into modes of both helicities. Thus, every time that $Q^{s}$ reaches a maximum at the excitation of an electric or a magnetic mode, that maximum value passes on to $Q_{+}^{s}$ and $Q_{-}^{s}[50,51]$. For these cases, the dielectric antenna renders an achiral behavior, i.e. $C^{s}(\sqrt{0.9}) \sim 0$. Nonetheless, looking at $C^{s}$ in Figure 4 , it can be observed that the antenna can also render an achiral behavior off resonance. In this latter case, the achirality stems from a non-trivial condition on the different modes.

Another feature that can be observed in Fig. 4 is that $C^{s}(\alpha)=C(\alpha)$ for some special wavelengths. At these wavelengths, the scattered field maintains the exact chirality as the emitter. In other words, the antenna becomes transparent to the helicity of the emitted field. Let us note that the cause of this phenomenon is not always the same. For some cases, the particle does not modify the helicity content of an emitter because the particle is dual. Dual particles, by definition, preserve the helicity of light $[8,30,31,39,43,53]$. In the macroscopic approximation of Maxwell equations, a single sphere embedded in air is dual if and only if $\epsilon=\mu$. However, as it is discussed in [31], an approximate dual condition can be found for certain wavelengths by computing the minimum of an intrinsic helicity transfer function defined as [31, 32] :

$$
T(R, \epsilon, \mu)=\frac{\sum_{j}\left|a_{j}-b_{j}\right|^{2}}{\sum_{j}\left|a_{j}+b_{j}\right|^{2}}
$$

The logarithm of the transfer function for the antenna in consideration ( $R=100 \mathrm{~nm}$, silicon-made) $\log _{10}\left[T(R, \epsilon, \mu)^{-1}\right]$ is plotted in Fig.5(b) and is compared with $C^{s}(\alpha, R, \lambda)$ plotted in Fig.5(a). It can be seen that both functions have similar features but they are spectrally shifted. It is also observed that 

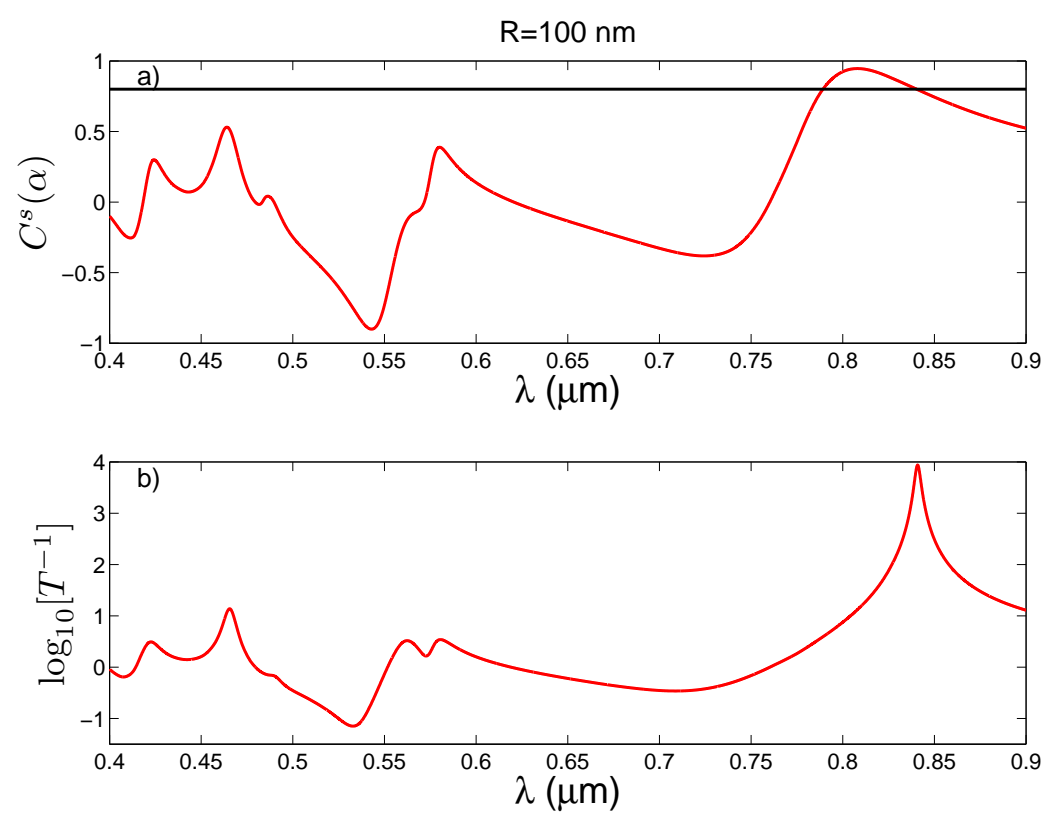

FIG. 5. Comparison between the chirality emitted by a Si-based resonator and its duality behavior. a) $C^{s}(\sqrt{0.9}, R, \lambda)$ in red and the initial $C(\sqrt{0.9})$ in black. b) $\log _{10}\left[T(R, \epsilon, \mu)^{-1}\right]$. Both plots have been done with a Si-made antenna of $R=100 \mathrm{~nm}$. All multipolar contributions are taken into account.

the antenna is only dual for $\lambda \approx 841 \mathrm{~nm}$, which corresponds to an almost zero of $T(R, \epsilon, \mu)$. This $\lambda$ clearly coincides with one of the intersection points between $C^{s}(\alpha)$ and $C(\alpha)$ in Fig.5. Nonetheless, the other intersection of $C^{s}(\alpha)$ and $C(\alpha)$ in Figure 5 at $\lambda \sim 780 \mathrm{~nm}$ does not correspond to a dual condition. That is, the condition $C^{s}(\alpha)=C(\alpha)$ can also occur without achieving duality. Yet there is a fundamental difference between the two $C^{s}(\alpha)=C(\alpha)$ conditions (dual and non-dual). The dual condition is an intrinsic property of the antenna, whereas the other one is not. That is, the wavelength at which the non-dual $C^{s}(\alpha)=C(\alpha)$ condition occurs depends on the emitter properties, such as the parameter $\alpha$. In contrast, the dual condition is fixed by the geometry and material of the antenna.

Since available mono-disperse colloidal solutions have $2 \%$ standard deviations [26], it is important to check if these changes in size could potentially spoil the behavior of the antenna. To verify that, we set out to study the value of $C^{s}(\alpha)$ for a radius of $R=(100 \pm 2) \mathrm{nm}$ around $\lambda=808 \mathrm{~nm}$, i.e. the wavelength at which $C^{s}(\alpha)$ has a maximum for $R=100 \mathrm{~nm}$. The results are depicted in Fig.6. It is observed that the 


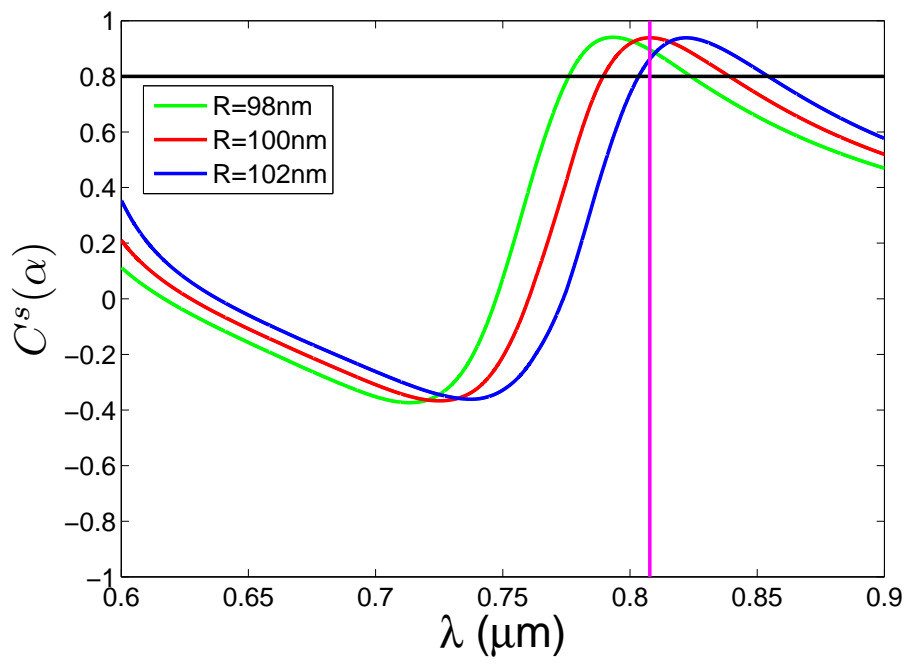

FIG. 6. Chirality dependence on the size of the antenna. The black horizontal line represents the initial chirality of the molecule $C(\alpha=\sqrt{0.9})=0.8$. In red, the scattered chirality $C^{s}(\alpha=\sqrt{0.9}, R=100)$. The vertical magenta line highlights the wavelength at which $C^{s}$ reaches a maximum. The green and blue lines are obtained using particles with $R=98,102 \mathrm{~nm}$ respectively.

chirality of the emitter is boosted for all the radius in the interval. Now, if the emitter happens to emit at a different wavelength, the antenna would have to be designed so that the maximum of $C^{s}(\alpha)$ happens at that wavelength. This has been quantified in the Appendix B, where it is shown that the chirality can be boosted for wavelengths ranging from the UV to the IR.

Last but not least, the differences in behavior between the gold and silicon antennas are summarized in Fig.7. We have carried out the simulations for a chiral emitter with $|\alpha|^{2}=0.9$, at a 10 nm distance from the surface of the antenna along the $z$ axis, oriented along the $z$ axis, and emitting at $\lambda=808 \mathrm{~nm}$. Their spectral behavior is shown at the bottom of Fig.7, where the vertical magenta line indicates the wavelength of the emission at $808 \mathrm{~nm}$. Then, at the top, we compute $\left|\mathbf{E}^{\mathrm{S}}\right|^{2}$ on a XY plane at $600 \mathrm{~nm}$ from the center of the resonator. It is observed, that while the Si antenna scatters all light in the +1 helicity component, the gold nano-antenna scatters almost evenly between the two helicity components. As for the scattered power, at this particular wavelength, it is seen that the two helicity components add up to a similar amount. 

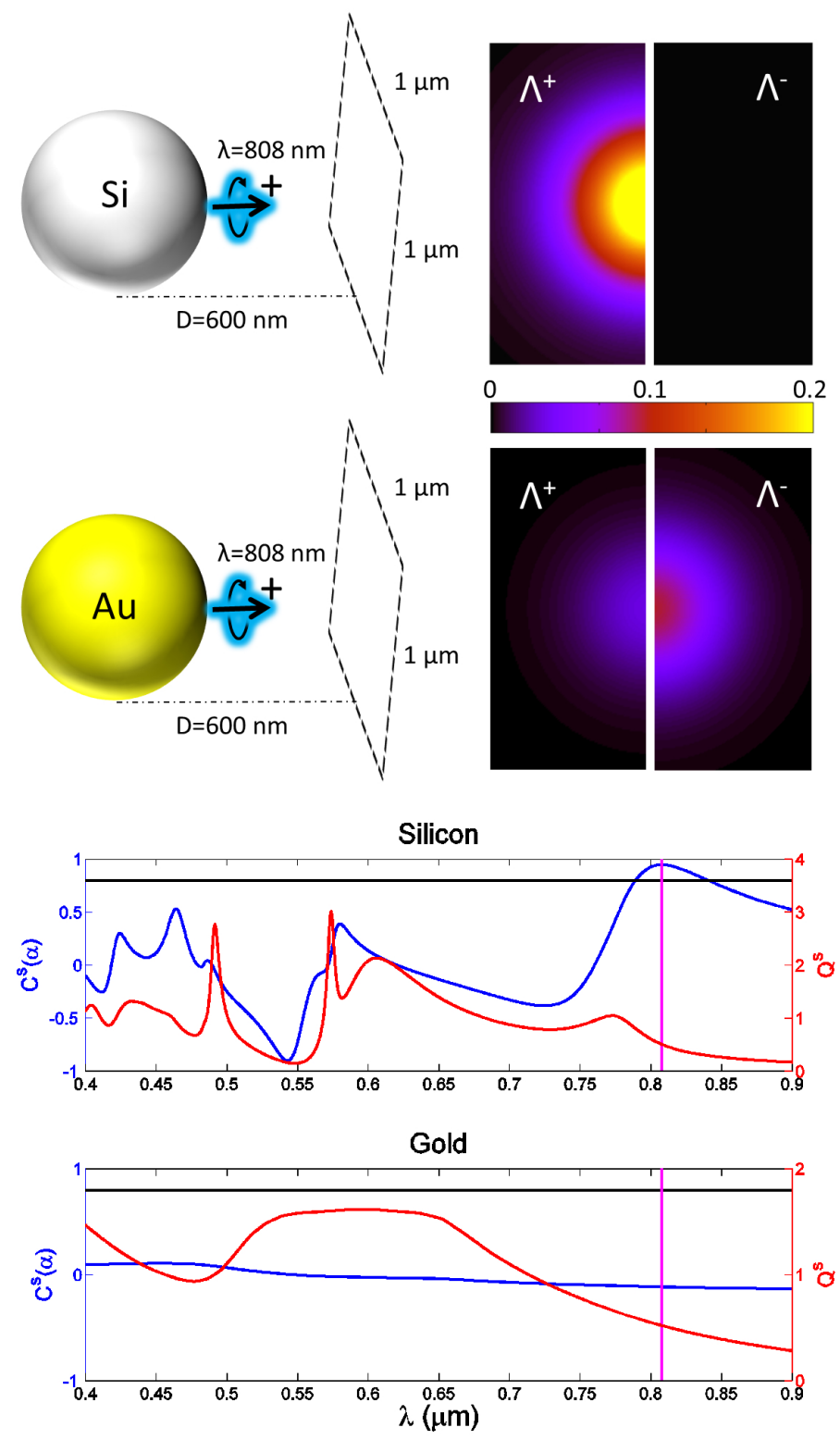

FIG. 7. A dipolar chiral emitter with $|\alpha|^{2}=0.9$ is placed at $10 \mathrm{~nm}$ of the surface of a spherical resonator with $R=100 \mathrm{~nm}$. Light is emitted at $\lambda=808 \mathrm{~nm}$ and the emitter is oriented along the $z$ axis. The scattered field is measured at a XY plane at $600 \mathrm{~nm}$ of the center of the resonator. The scattered field is projected into its two helicity components. a) The resonator is made of silicon. Most of the light is scattered in the +1 helicity component. b) The resonator is made of gold. The light is scattered almost equally in both helicity components. c) Spectral plots of $C^{s}(\alpha)$ and $Q^{s}$ for both resonators. The initial chirality of the emitter is also plotted as a black horizontal line. At 808nm (magenta vertical line), the Si resonator has $C_{S i}^{s}(\alpha) \approx 1$ boosting the initial chirality of the emitter, whereas $C_{A u}^{s}(\alpha) \approx 0$ yielding an almost achiral scattered field. 


\section{CONCLUSION}

We have presented a formulation based on the multipolar theory developed in the helicity basis to quantify the chirality of the field emitted by a system composed of a chiral dipole coupled to an optical antenna. First, it has been seen that gold spherical antennas do not feature the ability to tailor the chirality of emitted fields since they yield the same achiral response in the whole visible/near infrared range. This is due to the fact that plasmonic spherical nanoparticles behave mainly as electric dipoles. A very different feature was observed with silicon-made spherical nanoantennas. It has been shown that they are highly efficient to manipulate chiral fields emitted by dipolar emitters. They can yield scattered fields which almost encompass all the possible values of chirality, from $C^{s}=-1$ to $C^{s}=+1$ (see Fig.7). Given an emitter with an intrinsic chirality, the antenna can boost or reduce the chirality in the far field region. In particular, the antenna can yield a field with the exact same chirality of the emitter at the dual condition, and it can also yield completely achiral fields at the electric/magnetic resonant conditions. This result shows that transparent mono-disperse colloidal solutions of silicon particles could potentially be used to produce strong chiral effects with emitters.

\section{ACKNOWLEDGMENTS}

This work has been carried out thanks to the support of the A*MIDEX project $\left(\mathrm{n}^{\circ}\right.$ ANR-11-IDEX-000102) funded by the Investissements d'Avenir French Government program managed by the French National Research Agency (ANR).

\section{Appendix A}

In this section, we lay out the analytic formalism that will be used to compute the light-matter interaction between a single chiral emitter and a spherical resonator. Due to the symmetries of the problem, we will solve the problem using Mie theory. Mie theory is usually expressed in the electric/magnetic (or parity) basis. Nonetheless, in order to deal with chirality problems, it is more convenient to re-express the problem 
in the helicity basis $[30-32,45,46]$. Here, we will solve the Mie problem in the helicity basis to study the chirality of the field scattered by the spherical antenna.

The multipolar fields in the parity basis are well-known in optics [54-56]. We denote them as $\left\{\mathbf{A}_{j m_{z}}^{(e)}, \mathbf{A}_{j m_{z}}^{(m)}\right\}$. In contrast, the multipolar fields in the helicity basis are not very frequently used. They are linear combinations of electric and magnetic multipoles [30, 46]:

$$
\mathbf{A}_{j m_{z}}^{+}=\frac{\mathbf{A}_{j m_{z}}^{(m)}+i \mathbf{A}_{j m_{z}}^{(e)}}{\sqrt{2}} \quad \mathbf{A}_{j m_{z}}^{-}=\frac{\mathbf{A}_{j m_{z}}^{(m)}-i \mathbf{A}_{j m_{z}}^{(e)}}{\sqrt{2}}
$$

As a result, $\mathbf{A}_{j m_{z}}^{+}$and $\mathbf{A}_{j m_{z}}^{-}$are eigenstates of the helicity operator $\Lambda$ :

$$
\Lambda \mathbf{A}_{j m_{z}}^{+}=+\mathbf{A}_{j m_{z}}^{+} \quad \Lambda \mathbf{A}_{j m_{z}}^{-}=-\mathbf{A}_{j m_{z}}^{-}
$$

Notice that for both families of multipoles, the sub-indexes $j, m_{z}$ stand for the eigenvalues of the operators $\left(J^{2}\right)$ and $\left(J_{z}\right)$ [32]. Now, in order to solve the Mie problem (either in the parity or helicity basis), the decomposition of the incident field as a sum of multipoles needs to be found [57]. This will be the objective of the next section for the field emitted by the chiral emitter $\mathcal{C}$.

\section{The incident field}

Let us consider the field emitted by the chiral emitter $\mathcal{C}$ at a given wavelength $\lambda_{0}$. We will express the field with respect to a system of coordinates located on the emitter. Then, in the same way that the electric field emitted by an electric dipole $\mathbf{p}$ is given by an electric multipole of order $j=1$, the electric field produced by $\mathbf{p}_{+}$is also given by a multipole of order $j=1$ whose helicity content is +1 . Now, it has been assumed that the emitter does not emit as a pure dipole $\mathbf{p}_{+}$, but rather as $\mathbf{p}_{+}$with a probability of $|\alpha|^{2}$ and as $\mathbf{p}_{-}$ with a probability of $\left(1-|\alpha|^{2}\right)$. Hence, the electric field created by $\mathcal{C}$ will be:

$$
\mathbf{E}^{\mathcal{C}}(\mathbf{r})=\alpha \mathbf{A}_{1, m_{z}}^{+}+\sqrt{1-|\alpha|^{2}} \mathbf{A}_{1, m_{z}}^{-}
$$

In general, eq.(A.3) could be a summation over different values of $m_{z}$, depending on the orientation of the dipoles of the emitter. Here, to make calculations simpler, we will suppose that $m_{z}$ has a fixed value $m_{z}^{*}$, 
which has to be either $-1,0,+1$. Also notice that, due to their emitting behavior, the radial dependence of the multipolar fields in Eq.(A.3) are given by a spherical Hankel function $h_{1}\left(2 \pi r / \lambda_{0}\right)$.

As mentioned earlier, $\mathbf{E}^{\mathcal{C}}$ is the electric field created by $\mathcal{C}$ expressed with respect to the frame of reference located at the center of the emitter. However, to solve the Mie problem, we need to express this field with respect to a frame of reference located at the center of the antenna. Let $\mathbf{r}_{\mathbf{0}}$ be the position of the emitter with respect to the center of the sphere. Then, the expression of $\mathbf{E}^{\mathcal{C}}\left(\mathbf{r}-\mathbf{r}_{\mathbf{0}}\right)$ in terms of multipoles can be found by using the so-called translation-addition formulas [55, p.171][58, App.C][59]. Here, we will make two assumptions. First, without losing any generality, we will consider that the emitter is located along the $z$ axis, i.e. the position of the antenna $\mathbf{r}_{\mathbf{0}}=r_{0} \hat{\mathbf{z}}$. Second, we will consider that the emitter is located outside of the spherical antenna in consideration. Then, $\mathbf{E}^{\mathcal{C}}\left(\mathbf{r}-\mathbf{r}_{\mathbf{0}}\right)$ is expressed as:

$$
\mathbf{E}^{\mathcal{C}}\left(\mathbf{r}-r_{0} \hat{\mathbf{z}}\right)=\left\{\begin{array}{c}
\sum_{j^{\prime}=1}^{\infty} \sum_{L=0}^{\infty}\left[\alpha T_{j^{\prime}, L, 1, m_{z}^{*}} \operatorname{Rg}\left\{\mathbf{A}_{j^{\prime}, m_{z}^{*}}^{+}\right\}+\sqrt{1-|\alpha|^{2}} T_{j^{\prime}, L,-1, m_{z}^{*}} \operatorname{Rg}\left\{\mathbf{A}_{j^{\prime}, m_{z}^{*}}^{-}\right\}\right] \text {if }|\mathbf{r}|<r_{0}, \\
\sum_{j^{\prime}=1}^{\infty} \sum_{L=0}^{\infty}\left[\alpha \operatorname{Rg}\left\{T_{j^{\prime}, L, 1, m_{z}^{*}}\right\} \mathbf{A}_{j^{\prime}, m_{z}^{*}}^{+}+\sqrt{1-|\alpha|^{2}} \operatorname{Rg}\left\{T_{j^{\prime}, L,-1, m_{z}^{*}}\right\} \mathbf{A}_{j^{\prime}, m_{z}^{*}}^{-}\right] \text {if }|\mathbf{r}|>r_{0} .
\end{array}\right.
$$

where $T_{j^{\prime}, L, p, m_{z}}$ is defined as:

$$
T_{j^{\prime}, L, p, m_{z}}=(2 L+1)(-i)^{L} h_{L}\left(\frac{2 \pi r_{0}}{\lambda_{0}}\right)\left\langle m_{z}, 0\left(j^{\prime}, L\right) 1, m_{z}\right\rangle\left\langle 1, p\left(j^{\prime}, L\right) p, 0\right\rangle
$$

with $\left\langle J, M\left(j, j^{\prime}\right) m, m^{\prime}\right\rangle$ being the Clebsch-Gordan coefficients [55]. Note that it is assumed that both $T_{j^{\prime}, L, p, m_{z}}$ and $\mathbf{A}_{j m_{z}}^{ \pm}$have a radial dependence that is given by a Hankel function. Then, when a Bessel function dependence is needed, this is made explicit by the $\operatorname{Rg}\{\cdot\}$ sign, e.g. in eq.(A.4).

\section{The Mie problem}

Due to the symmetries of the Mie problem, once the multipolar decomposition of the incident field is known, the field scattered by the antenna immediately follows. Now, for this case, the incident field is $\mathbf{E}^{\mathcal{C}}\left(\mathbf{r}-r_{0} \hat{\mathbf{z}}\right)$. However, $\mathbf{E}^{\mathcal{C}}\left(\mathbf{r}-r_{0} \hat{\mathbf{z}}\right)$ has a different multipolar decomposition if $|\mathbf{r}| \lessgtr r_{0}$. Here, we will use the expression of $\mathbf{E}^{\mathcal{C}}\left(\mathbf{r}-r_{0} \hat{\mathbf{z}}\right)$ for $|\mathbf{r}|=R$, with $R$ being the radius of the spherical antenna. As mentioned 
above, it will be assumed that the emitter is located outside the sphere, i.e. $r_{0}>R$. Then, the incident field used to solve the Mie problem will be:

$$
\mathbf{E}^{\mathrm{i}}(\alpha)=\sum_{j^{\prime}=1}^{\infty} \sum_{L=0}^{\infty}\left[\alpha T_{j^{\prime}, L, 1, m_{z}^{*}} \operatorname{Rg}\left\{\mathbf{A}_{j^{\prime}, m_{z}^{*}}^{+}\right\}+\sqrt{1-|\alpha|^{2}} T_{j^{\prime}, L,-1, m_{z}^{*}} \operatorname{Rg}\left\{\mathbf{A}_{j^{\prime}, m_{z}^{*}}^{-}\right\}\right]
$$

As it was shown in [30, 32], if the multipolar decomposition of the incident field is $\mathbf{E}^{\mathrm{i}}=\sum_{j, m_{z}} \alpha_{j m_{z}}^{p} \mathbf{A}_{j m_{z}}^{p}$, then the scattered and field inside of the sphere are given by:

$$
\begin{aligned}
\mathbf{E}^{\mathrm{S}} & =\sum_{j, m_{z}} \alpha_{j m_{z}}^{p}\left[\frac{a_{j}+b_{j}}{\sqrt{2}} \mathbf{A}_{j m_{z}}^{p}+\frac{a_{j}-b_{j}}{\sqrt{2}} \mathbf{A}_{j m_{z}}^{-p}\right] \\
\mathbf{E}^{\mathrm{int}} & =\sum_{j, m_{z}} \alpha_{j m_{z}}^{p}\left[\frac{d_{j}+c_{j}}{\sqrt{2}} \mathbf{A}_{j m_{z}}^{p}+\frac{d_{j}-c_{j}}{\sqrt{2}} \mathbf{A}_{j m_{z}}^{-p}\right]
\end{aligned}
$$

where $\left\{a_{j}, b_{j}, c_{j}, d_{j}\right\}$ are the so-called Mie coefficients [49]. Thus, using eqs.(A.6-A.7), we obtain that the emitter-antenna interaction results in the following scattered field:

$$
\begin{aligned}
& \mathbf{E}^{\mathrm{S}}(\alpha)=\sum_{j^{\prime}=1}^{\infty} \sum_{L=0}^{\infty}\left[\left(\alpha T_{j^{\prime}, L, 1, m_{z}^{*}} \frac{a_{j}+b_{j}}{\sqrt{2}}+\sqrt{1-|\alpha|^{2}} T_{j^{\prime}, L,-1, m_{z}^{*}} \frac{a_{j}-b_{j}}{\sqrt{2}}\right) \mathbf{A}_{j^{\prime}, m_{z}^{*}}^{+}\right. \\
& \left.+\left(\alpha T_{j^{\prime}, L, 1, m_{z}^{*}} \frac{a_{j}-b_{j}}{\sqrt{2}}+\sqrt{1-|\alpha|^{2}} T_{j^{\prime}, L,-1, m_{z}^{*}} \frac{a_{j}+b_{j}}{\sqrt{2}}\right) \mathbf{A}_{j^{\prime}, m_{z}^{*}}^{-}\right]
\end{aligned}
$$

Eq.(A.8) models the effect that the antenna has on the light emitted by $\mathcal{C}$. In order to quantify the effects of the antenna on a chirality measurement, we look at the ratio of the two helicity component of the scattered power. Due to the orthogonality between the modes of helicity + and - , we can separate the total scattered power $\mathrm{W}^{\mathrm{s}}$ as the addition of two terms with a well-defined helicity, i.e. $\mathrm{W}^{\mathrm{s}}=\mathrm{W}_{+}^{\mathrm{s}}+\mathrm{W}_{-}^{\mathrm{s}}$. Each of these terms is computed as:

$$
\begin{aligned}
& \mathrm{W}_{+}^{\mathrm{s}}=\sum_{j^{\prime}, L}\left|\alpha T_{j^{\prime}, L, 1, m_{z}^{*}} \frac{a_{j}+b_{j}}{\sqrt{2}}+\sqrt{1-|\alpha|^{2}} T_{j^{\prime}, L,-1, m_{z}^{*}} \frac{a_{j}-b_{j}}{\sqrt{2}}\right|^{2} \\
& \mathrm{~W}_{-}^{\mathrm{s}}=\sum_{j^{\prime}, L}\left|\alpha T_{j^{\prime}, L, 1, m_{z}^{*}} \frac{a_{j}-b_{j}}{\sqrt{2}}+\sqrt{1-|\alpha|^{2}} T_{j^{\prime}, L,-1, m_{z}^{*}} \frac{a_{j}+b_{j}}{\sqrt{2}}\right|^{2}
\end{aligned}
$$

Both $\mathrm{W}^{\mathrm{s}}$ and $\mathrm{W}_{ \pm}^{\mathrm{s}}$ are given in power units. Then, in order to give dimensionless results for the scattering, scattering efficiency factors $Q^{s}$ have been computed throughout the manuscript. These are computed as the flux of the Poynting vector across a spherical surface, divided over the size parameter:

$$
Q^{s}=Q_{+}^{s}+Q_{-}^{s}=\frac{\mathrm{W}^{\mathrm{s}} \lambda^{2}}{4 \pi^{2} R^{2}}=\frac{\left(\mathrm{W}_{+}^{\mathrm{s}}+\mathrm{W}_{-}^{\mathrm{s}}\right) \lambda^{2}}{4 \pi^{2} R^{2}}
$$




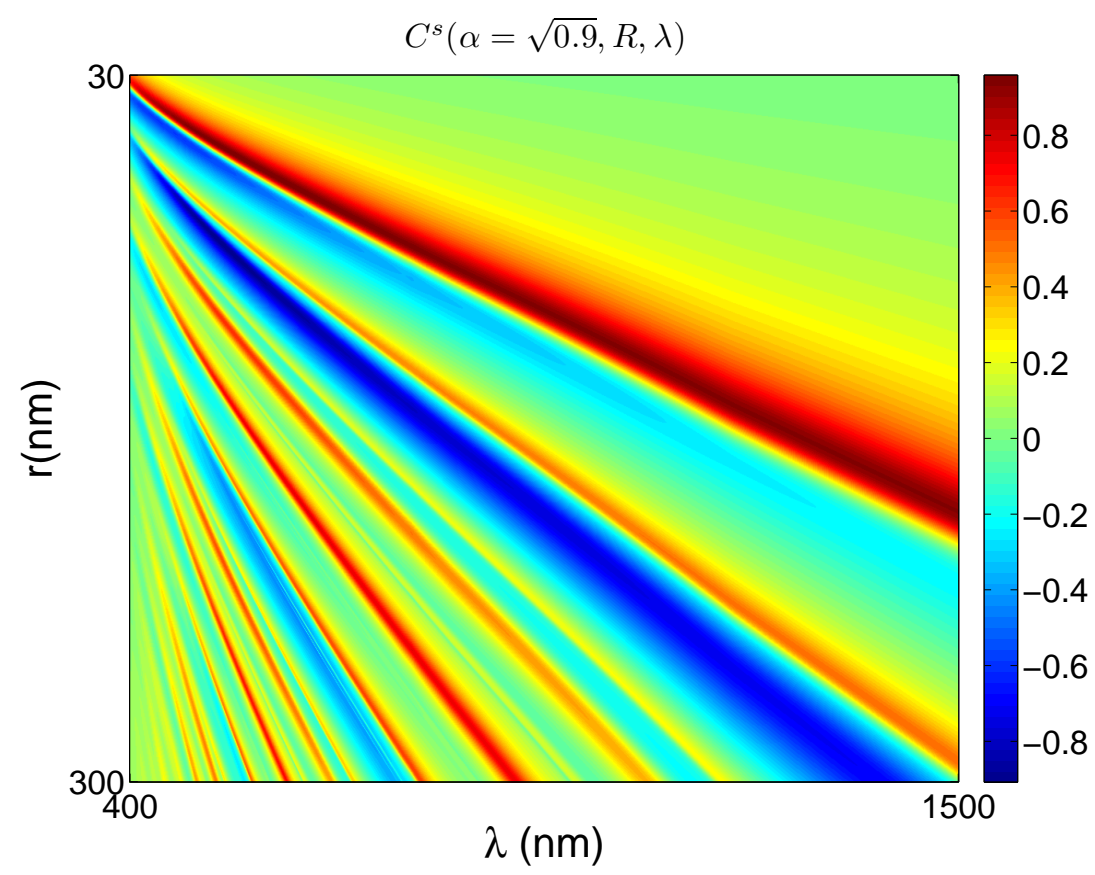

FIG. 8. $C^{s}(\alpha=\sqrt{0.9}, R, \lambda)$ for $R=30-300 \mathrm{~nm}$ and $\lambda=400-900 \mathrm{~nm}$. The antenna is made of Si.

Finally, in the same way as we did for the emitter in Eq.(3), we can define the induced chirality of the antenna as:

$C^{s}(\alpha)=\frac{\sum_{j^{\prime}, L}\left|\alpha T_{j^{\prime}, L, 1, m_{z}} \frac{a_{j}+b_{j}}{\sqrt{2}}+\sqrt{1-|\alpha|^{2}} T_{j^{\prime}, L,-1, m_{z}} \frac{a_{j}-b_{j}}{\sqrt{2}}\right|^{2}-\left|\alpha T_{j^{\prime}, L, 1, m_{z}} \frac{a_{j}-b_{j}}{\sqrt{2}}+\sqrt{1-|\alpha|^{2}} T_{j^{\prime}, L,-1, m_{z}} \frac{a_{j}+b_{j}}{\sqrt{2}}\right|^{2}}{\sum_{j^{\prime}, L}\left|\alpha T_{j^{\prime}, L, 1, m_{z}} \frac{a_{j}+b_{j}}{\sqrt{2}}+\sqrt{1-|\alpha|^{2}} T_{j^{\prime}, L,-1, m_{z}} \frac{a_{j}-b_{j}}{\sqrt{2}}\right|^{2}+\left|\alpha T_{j^{\prime}, L, 1, m_{z}} \frac{a_{j}-b_{j}}{\sqrt{2}}+\sqrt{1-|\alpha|^{2}} T_{j^{\prime}, L,-1, m_{z}} \frac{a_{j}+b_{j}}{\sqrt{2}}\right|^{2}}$

Eq.(A.12) is the main analytic result of this paper. Given the chirality of the emitter $C(\alpha), C^{s}(\alpha)$ predicts the induced chirality by the spherical antenna. Note that $C^{s}(\alpha)$ is a multi-variable function. Not only does it depend on $\alpha$, but it is also a function of other properties of the emitter such as $\lambda_{0}, m_{z}^{*}, r_{0}$. In addition, $C^{s}(\alpha)$ depends on some parameters of the antenna, $\epsilon, \mu, R$.

\section{APPENDIX B}

In this section, we show that even though the study above has been done with small resonators, larger antennas do not improve the chirality features shown in Fig.3. Using eq.(5), we can compute the chirality of the scattering arbitrarily modifying the radius $R$ of the antenna and the $\lambda$ of the emission. The results 

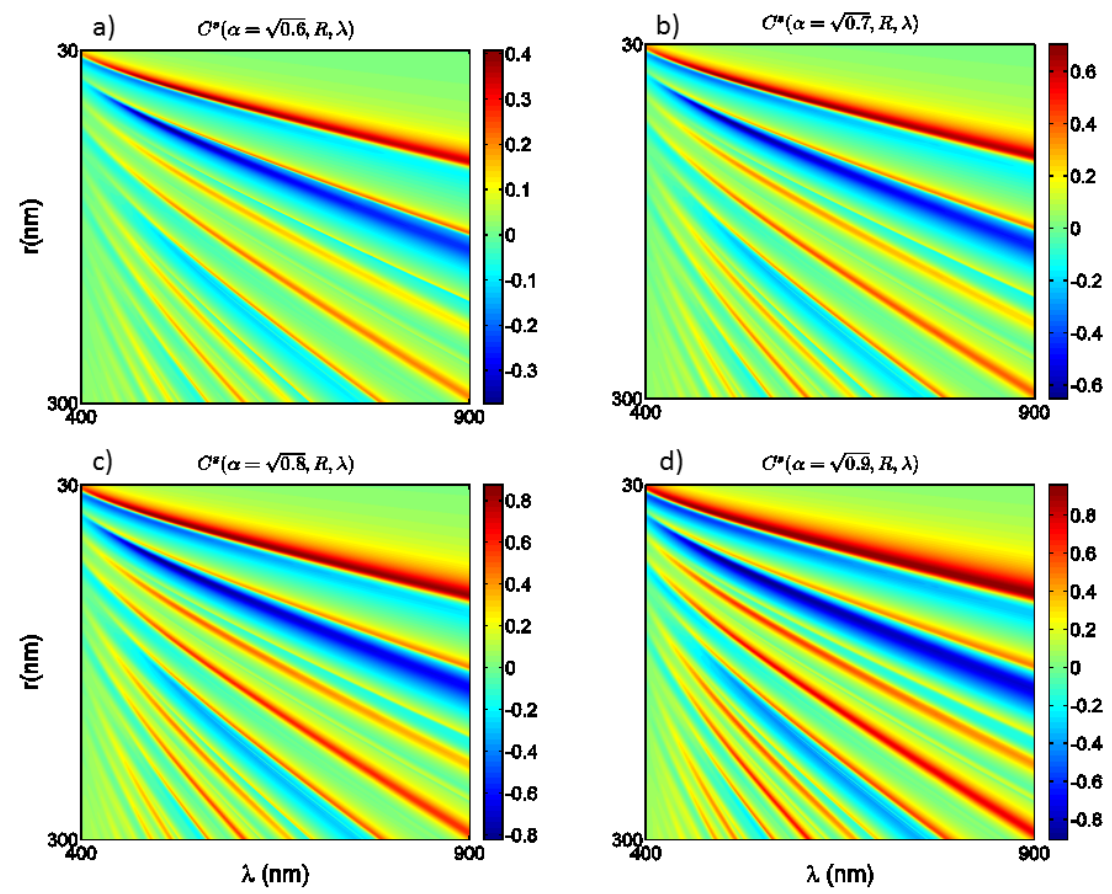

FIG. 9. Influence of the initial chirality of the emitter on the chirality of the scattered field by the antenna. $C^{s}(\alpha, R, \lambda)$ for $R=30-300 \mathrm{~nm}$ and $\lambda=400-900 \mathrm{~nm}$ is plotted for a) $\alpha=\sqrt{0.6}$, b) $\alpha=\sqrt{0.7}$, c) $\alpha=\sqrt{0.8}, \alpha=\sqrt{0.9}$. The antenna is made of $\mathrm{Si}$.

are displayed in Fig.8 for $R=30-300 \mathrm{~nm}, \lambda=400-1500 \mathrm{~nm}$, and $|\alpha|^{2}=0.9$. Fig.8 demonstrates that small antennas achieve a larger range of chirality values in the visible domain. For telecommunication wavelengths, similar chirality modulations are achieved, but the features of the antenna are spectrally widened. Now, as it is numerically shown next, the largest chirality modulations are consistently given in the intervals $R=50-100 \mathrm{~nm}$ and $450-600 \mathrm{~nm}$. Actually, this fact is shown to be independent of the initial chirality of the emitter, modeled by $\alpha$, even though $\alpha$ plays a quantitative role. To demonstrate this, in Fig. 9 , we depict $C^{s}(\alpha, R, \lambda)$ for $|\alpha|^{2}=0.6,0.7,0.8,0.9$ and $R=30-300 \mathrm{~nm}$ and $\lambda=400-900 \mathrm{~nm}$. The antenna is made of Si. It can be seen that the shape of all the $(R, \lambda)$ plots resemble each other. In contrast, the accessible values for $C^{s}(\alpha)$, given by the colorbar, vary from one plot to the other. Actually, it is seen that the greater $\alpha$ is, the larger interval of chirality values the antenna can achieve.

Moreover, it is observed that most of the interesting phenomena occur for $R \in[50-100] \mathrm{nm}$ and $\lambda \in$ 

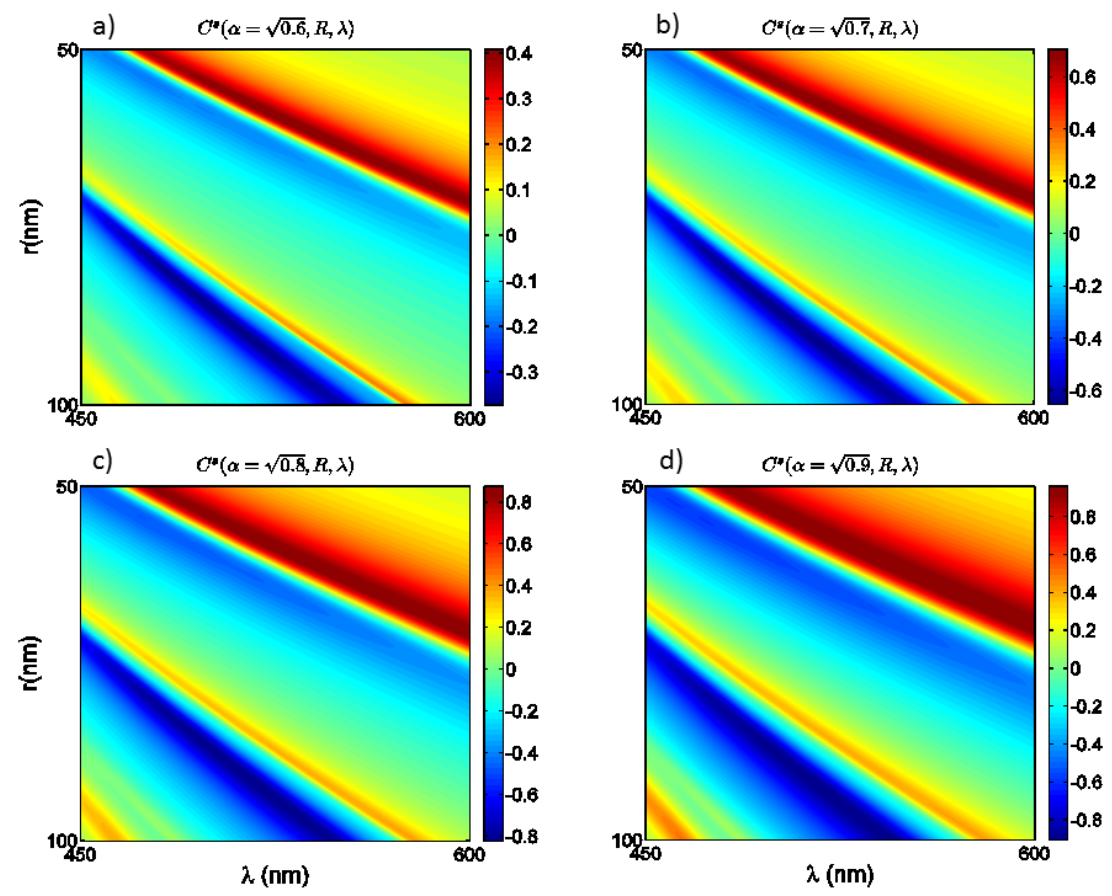

FIG. 10. Influence of the initial chirality of the emitter on the chirality of the scattered field by the antenna. $C^{s}(\alpha, R, \lambda)$ for $R=50-100 \mathrm{~nm}$ and $\lambda=450-600 \mathrm{~nm}$ is plotted for a) $\left.\left.\alpha=\sqrt{0.6}, \mathrm{~b}\right) \alpha=\sqrt{0.7}, \mathrm{c}\right) \alpha=\sqrt{0.8}$, $\alpha=\sqrt{0.9}$. The antenna is made of $\mathrm{Si}$.

$[450-600] \mathrm{nm}$. These phenomena encompass inversion and boost of the initial chirality of the emitter, as well as dual and achiral behaviors. Thus, in Figure 10 we show $C^{s}(\alpha, R, \lambda)$ for 4 different $|\alpha|^{2}=0.6,0.7,0.8,0.9$ and $R=50-100 \mathrm{~nm}$ and $\lambda=450-600 \mathrm{~nm}$. Judging by the colorbars, it is seen that all the accessible chirality states are encompassed in this range.

*xavislow@protonmail.com

$\dagger$ nicolas.bonod@fresnel.fr

[1] D. Punj, M. Mivelle, S. B. Moparthi, T. S. van Zanten, H. Rigneault, N. F. van Hulst, M. F. García-Parajó and J. Wenger, Nature nanotechnology, 2013, 8, 512-516.

[2] A. Puchkova, C. Vietz, E. Pibiri, B. Wnsch, M. Sanz Paz, G. P. Acuna and P. Tinnefeld, Nano letters, 2015, 15, $8354-8359$. 
[3] M. P. Busson, B. Rolly, B. Stout, N. Bonod and S. Bidault, Nature Communications, 2012, 3, 962-.

[4] G. Acuna, F. Möller, P. Holzmeister, S. Beater, B. Lalkens and P. Tinnefeld, Science, 2012, 338, 506-510.

[5] T. B. Hoang, G. M. Akselrod and M. H. Mikkelsen, Nano letters, 2016, 16, 270275.

[6] M. W. Knight, H. Sobhani, P. Nordlander and N. J. Halas, Science, 2011, 332, 702-704.

[7] A. García-Etxarri and J. A. Dionne, Phys. Rev. B, 2013, 87, 235409.

[8] I. Fernandez-Corbaton, M. Fruhnert and C. Rockstuhl, ACS Photonics, 2015, 2, 376-384.

[9] M. Decker, M. W. Klein, M. Wegener and S. Linden, Opt. Lett., 2007, 32, 856-858.

[10] M. Schäferling, D. Dregely, M. Hentschel and H. Giessen, Physical Review X, 2012, 2, 1-9.

[11] A. Kuzyk, R. Schreiber, Z. Fan, G. Pardatscher, E.-M. Roller, A. Högele, F. C. Simmel, A. O. Govorov and T. Liedl, Nature, 2012, 483, 311-314.

[12] R. Schreiber, N. Luong, Z. Fan, A. Kuzyk, P. C. Nickels, T. Zhang, D. M. Smith, B. Yurke, W. Kuang, A. O. Govorov et al., Nature communications, 2013, 4, 2948.

[13] X. Vidal, W. J. Kim, A. Baev, V. Tokar, H. Jee, M. T. Swihart and P. N. Prasad, Nanoscale, 2013, 5, 1055010555.

[14] X. Shen, P. Zhan, A. Kuzyk, Q. Liu, A. Asenjo-Garcia, H. Zhang, F. J. G. de Abajo, A. Govorov, B. Ding and N. Liu, Nanoscale, 2014, 6, 2077-2081.

[15] X. Duan, S. Yue and N. Liu, Nanoscale, 2015, 7, 17237-17243.

[16] W. Löffler, D. J. Broer and J. P. Woerdman, Phys. Rev. A, 2011, 83, 065801.

[17] D. L. Andrews and M. M. Coles, Opt. Lett., 2012, 37, 3009-3011.

[18] Y. Gorodetski, A. Drezet, C. Genet and T. W. Ebbesen, Phys. Rev. Lett., 2013, 110, 203906.

[19] K. Y. Bliokh, A. Y. Bekshaev and F. Nori, New Journal of Physics, 2013, 15, 033026.

[20] X. Zambrana-Puyalto, X. Vidal and G. Molina-Terriza, Nat. Commun., 2014, 5, 4922.

[21] S. S. Kruk, M. Decker, I. Staude, S. Schlecht, M. Greppmair, D. N. Neshev, and Y. S. Kivshar, ACS Photon., $2014, \mathbf{1}, 1218-1223$.

[22] X. Zambrana-Puyalto, X. Vidal, I. Fernandez-Corbaton and G. Molina-Terriza, Sci. Rep., 2016, 6, 22185.

[23] M. P. Moloney, Y. K. Gun'ko and J. M. Kelly, Chemical Communications, 2007, 3900-3902.

[24] N. Suzuki, Y. Wang, P. Elvati, Z.-b. Qu, K. Kim, S. Jiang, E. Baumeister, J. Lee, B. Yeom, J. H. Bahng et al., ACS nano, 2016.

[25] J. M. Romo-Herrera, R. A. Alvarez-Puebla and L. M. Liz-Marzán, Nanoscale, 2011, 3, 1304-1315.

[26] L. Shi, J. T. Harris, R. Fenollosa, I. Rodriguez, X. Lu, B. A. Korgel and F. Meseguer, Nature Communications, 
2013, 4, 1904.

[27] A. B. Evlyukhin, S. M. Novikov, U. Zywietz, R. L. Eriksen, C. Reinhardt, S. I. Bozhevolnyi and B. N. Chichkov, Nano letters, 2012, 12, 3749-3755.

[28] A. I. Kuznetsov, A. E. Miroshnichenko, Y. H. Fu, J. Zhang and B. Lukyanchuk, Scientific reports, $2012,2,492$.

[29] U. Zywietz, A. B. Evlyukhin, C. Reinhardt and B. N. Chichkov, Nature Communications, $2014,5,3402$.

[30] X. Zambrana-Puyalto, I. Fernandez-Corbaton, M. L. Juan, X. Vidal and G. Molina-Terriza, Opt. Lett., 2013, 38, 1857-1859.

[31] X. Zambrana-Puyalto, X. Vidal, M. L. Juan and G. Molina-Terriza, Optics express, 2013, 21, 17520-17530.

[32] X. Zambrana-Puyalto, PhD thesis, Macquarie University, 2014.

[33] T. Davis and D. Gómez, Physical Review B, 2014, 90, 235424.

[34] V. Grigoriev, N. Bonod, J. Wenger and B. Stout, ACS Photonics, 2015, 2, 263-270.

[35] S. Yoo and Q.-H. Park, Scientific reports, 2015, 5, 14463.

[36] A. García-Etxarri, R. Gómez-Medina, L. S. Froufe-Pérez, C. López, L. Chantada, F. Scheffold, J. Aizpurua, M. Nieto-Vesperinas and J. J. Sáenz, Optics Express, 2011, 19, 4815-4826.

[37] B. Rolly, B. Bebey, S. Bidault, B. Stout and N. Bonod, Physical Review B: Condensed Matter and Materials Physics, 2012, 85, 245432.

[38] X. Zambrana-Puyalto and N. Bonod, Phys. Rev. B, 2015, 91, 195422.

[39] I. Fernandez-Corbaton, X. Zambrana-Puyalto, N. Tischler, X. Vidal, M. L. Juan and G. Molina-Terriza, Phys. Rev. Lett., 2013, 111, 060401.

[40] J. A. Schellman, Chemical reviews, 1975, 75, 323.

[41] D. P. Craig and T. Thirunamachandran, Molecular quantum electrodynamics: an introduction to radiationmolecule interactions, Courier Corporation, 1998.

[42] I. Fernandez-Corbaton, X. Vidal, N. Tischler and G. Molina-Terriza, The Journal of Chemical Physics, 2013, 138, 214311.

[43] I. Fernandez-Corbaton and C. Rockstuhl, arXiv preprint arXiv:1508.04049, 2015.

[44] X. Vidal, I. Fernandez-Corbaton, A. F. Barbara and G. Molina-Terriza, Applied Physics Letters, 2015, 107, 211107.

[45] I. Fernandez-Corbaton, PhD thesis, Macquarie University, 2014.

[46] I. Fernandez-Corbaton, X. Zambrana-Puyalto and G. Molina-Terriza, Phys. Rev. A, 2012, 86, 042103.

[47] I. Fernandez-Corbaton, S. Nanz, R. Alaee and C. Rockstuhl, Optics Express, 2015, 23, 33044-33064. 
[48] E. Palik and G. Ghosh, Handbook of optical constants of solids, Academic Press, Boston, 1998.

[49] C. F. Bohren and D. R. Huffman, Absorption and scattering of light by small particles, Wiley, 1983.

[50] X. Zambrana-Puyalto, X. Vidal and G. Molina-Terriza, Opt. Express, 2012, 20, 24536-24544.

[51] N. Tischler, I. Fernandez-Corbaton, X. Zambrana-Puyalto, A. Minovich, X. Vidal, M. L. Juan and G. MolinaTerriza, Light Sci Appl, 2014, 3, e183.

[52] G. Vuye, S. Fisson, V. N. Van, Y. Wang, J. Rivory and F. Abeles, Thin Solid Films, 1993, 233, $166-170$.

[53] M. K. Schmidt, J. Aizpurua, X. Zambrana-Puyalto, X. Vidal, G. Molina-Terriza and J. J. Sáenz, Phys. Rev. Lett., 2015, 114, 113902.

[54] M. E. Rose, Elementary Theory of Angular Momentum, Wiley, New York, 1957.

[55] W.-K. Tung, Group Theory in Physics, World Scientific, Singapore, 1985.

[56] J. D. Jackson, Classical Electrodynamics : Third Edition, John Wiley \& Sons, New York, 1999.

[57] G. Gouesbet and G. Gréhan, Generalized Lorenz-Mie Theories, Springer, 2011.

[58] M. Mishchenko, L. Travis and A. Lacis, Scattering, Absorption, and Emission of Light by Small Particles, Cambridge University Press, 2002, p. 462.

[59] R. C. Wittmann, Antennas and Propagation, IEEE Transactions on, 1988, 36, 1078-1087. 

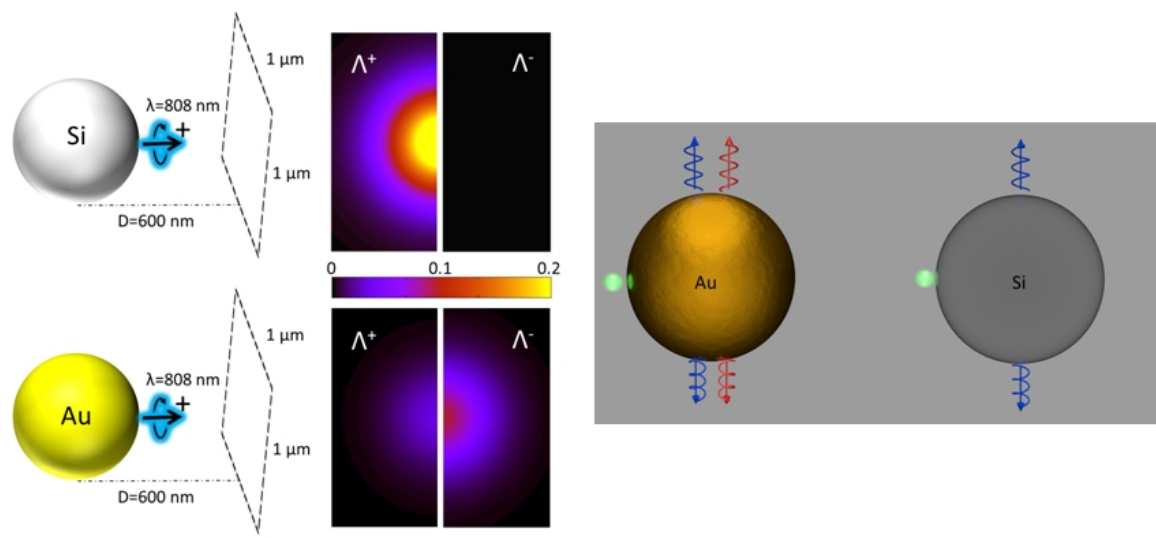

Graphical TOC entry 